\title{
Combined Mueller and Jones matrix method for the evaluation of the complex modulation in a liquid-crystal-on-silicon display
}

\author{
I. Moreno, ${ }^{1, *}$ A. Lizana, ${ }^{2}$ J. Campos, ${ }^{2}$ A. Márquez,${ }^{3}$ C. Iemmi, ${ }^{4}$ and M. J. Yzuel ${ }^{2}$ \\ ${ }^{1}$ Departamento de Ciencia de Materiales, Optica y Tecnología Electrónica, Universidad Miguel Hernández, \\ 03202 Elche, Spain \\ ${ }^{2}$ Departamento de Física, Universidad Autónoma de Barcelona, 08193 Bellaterra, Spain \\ ${ }^{3}$ Departamento de Física, Ingeniería de Sistemas y Teoría de la Señal, Universidad de Alicante, \\ 03080 Alicante, Spain \\ ${ }^{4}$ Departamento de Física, Facultad de Ciencias Exactas y Naturales, Universidad de Buenos Aires, \\ 1428 Buenos Aires, Argentina \\ *Corresponding author: i.moreno@umh.es
}

Received November 16, 2007; revised February 8, 2008; accepted February 12, 2008; posted February 19, 2008 (Doc. ID 89860); published March 14, 2008

\begin{abstract}
We apply the polar decomposition of the Mueller matrix describing a liquid-crystal-on-silicon display to identify the diattenuator, depolarizer, and retarder contributions as a function of the gray level. The retarder contribution is expressed in terms of the equivalent Jones matrix to apply previous techniques to evaluate the phase modulation. This allows searching for optimized polarization configurations for phase- or amplitude-only modulation responses. We present results for $\lambda=633 \mathrm{~nm}$ showing a phase-only modulation up to $2 \pi \mathrm{rad}$ and flat intensity modulation. (C) 2008 Optical Society of America

OCIS codes: $230.3720,230.6120,260.5430$.
\end{abstract}

Liquid crystal displays (LCDs) have found widespread use as spatial light modulators (SLMs) in applications such as optical image processing, holographic data storage, programmable adaptive and diffractive optics, and optical metrology. For all these applications, their configuration as phase-only SLMs is very important to obtain high values of the diffraction efficiency [1]. This situation is achieved when the input and output polarization configurations are properly selected [2]. In recent years new liquidcrystal-on-silicon (LCoS)-based displays have attracted considerable interest due to their high resolution and excellent fill factor [3]. They are reflective displays that, due to the double pass through the device, allow for an increased phase modulation depth compared to previous twisted nematic (TN) LCDs. Several methods have been proposed to evaluate and predict the phase modulation for different polarization configurations. They usually employ the Jones matrix formalism $[4,5]$, since the complex (amplitude and phase) modulation can be directly evaluated. However, the Jones formalism does not account for such other important polarimetric parameters as diattenuation and depolarization. For that purpose, the Mueller matrix formalism provides an alternative description. This approach is especially relevant to show evidence of a certain degree of depolarized light in the displays [6-8]. The Mueller approach has also been used to find LCD configurations leading to a phase-only modulation. For instance, Pezzaniti and Chipman [9] measured the Mueller matrix of a TNLCD and calculated its average eigenpolarization states. More recently, the trajectory on the Poincaré sphere and its modification with external wave plates has also been employed with a TN-LCD [10]. These methods very elegantly and efficiently lead to a constant intensity configuration, but the phase modulation cannot be evaluated $a$ priori and has to be mea- sured. However, the possibility of predicting the phase-modulation is very desirable, since the phasemodulation depth can be increased in configurations with flat but no maximum intensity modulation [11].

In this work we combine the Mueller and Jones approaches to perform a complete characterization of a LCoS display. We use results of a previous polarimetric characterization of the display based on the Mueller matrix approach [8], which showed up to $10 \%$ of the depolarized light for some gray levels and for certain incident states of polarization. In this situation, previous Jones matrix approaches to obtain a phaseonly response $[4,5]$ are not directly applicable. However, the use of the Jones formalism is still of interest to predict the phase modulation with good accuracy. For that purpose we apply the polar decomposition [12] of the measured Mueller matrix. This decomposition states that a general Mueller matrix $\mathbf{M}$ can be written as the product of the three factors of diattenuation, retardance, and depolarization as $\mathbf{M}$ $=\mathbf{M}_{\Delta} \mathbf{M}_{\mathbf{R}} \mathbf{M}_{\mathbf{D}}$. In our previous work [8] we showed that the diattenuation was negligible, so the polar decomposition $\mathbf{M}=\mathbf{M}_{\Delta} \mathbf{M}_{\mathbf{R}}$ can be applied as [12]

$$
\mathbf{M}=\left(\begin{array}{cc}
1 & \overrightarrow{0}^{T} \\
\vec{P}_{\Delta} & m_{\Delta}
\end{array}\right)\left(\begin{array}{cc}
1 & \overrightarrow{0}^{T} \\
\overrightarrow{0} & m_{R}
\end{array}\right)=\left(\begin{array}{cc}
1 & \overrightarrow{0}^{T} \\
\vec{P}_{\Delta} & m_{\Delta} m_{R}
\end{array}\right) .
$$

Here $\mathbf{M}_{\Delta}$ and $\mathbf{M}_{\mathbf{R}}$ are, respectively, the depolarization and retardance matrices, $\overrightarrow{0}^{T}=\left(\begin{array}{lll}0 & 0 & 0\end{array}\right) ; \vec{P}_{\Delta}$ is the polarizance vector; and $m_{\Delta}$ and $m_{R}$ are $3 \times 3$ submatrices that can be calculated using the procedure described in [12]. Once $\mathbf{M}_{\mathbf{R}}$ has been calibrated, the equivalent Jones matrix can be calculated and used for the evaluation of the phase modulation. For that purpose we follow the technique proposed in [5], since it can be applied with a single wavelength and no assumptions are required about the microscopic model for 
the liquid crystal (LC) distribution in the LC cell. This technique considers that any nonabsorbing reciprocal polarization device (for which the LCD is a particular case) can be described by a unimodular unitary Jones matrix, i.e.,

$$
\mathbf{J}_{\mathbf{R}}=e^{-i \beta}\left(\begin{array}{cc}
A & B \\
-B^{*} & A^{*}
\end{array}\right) .
$$

Here $A=A_{\mathrm{Re}}-i A_{\mathrm{Im}}$ and $B=B_{\mathrm{Re}}-i B_{\mathrm{Im}}$ are complex parameters (Re and Im denote the real and imaginary parts) that depend on the addressed voltage and fulfill the condition $|A|^{2}+|B|^{2}=1$. The retarder Mueller matrix equivalent to the Jones matrix in Eq. (2) is

$$
\mathbf{M}_{\mathbf{R}}=\left(\begin{array}{cccc}
1 & 0 & 0 & 0 \\
0 & |A|^{2}-|B|^{2} & 2\left(A_{\mathrm{Re}} B_{\mathrm{Re}}-A_{\mathrm{Im}} B_{\mathrm{Im}}\right) & 2\left(A_{\mathrm{Im}} B_{\mathrm{Re}}-A_{\mathrm{Re}} B_{\mathrm{Im}}\right) \\
0 & -2\left(A_{\mathrm{Re}} B_{\mathrm{Re}}-A_{\mathrm{Im}} B_{\mathrm{Im}}\right) & A_{\mathrm{Re}}^{2}-A_{\mathrm{Im}}^{2}+B_{\mathrm{Re}}^{2}-B_{\mathrm{Im}}^{2} & 2 A_{\mathrm{Re}} A_{\mathrm{Im}}+2 B_{\mathrm{Re}} B_{\mathrm{Im}} \\
0 & 2\left(A_{\mathrm{Re}} B_{\mathrm{Im}}+A_{\mathrm{Im}} B_{\mathrm{Re}}\right) & -2 A_{R} A_{I}-2 B_{R} B_{I} & A_{\mathrm{Re}}^{2}-A_{\mathrm{Im}}^{2}-B_{\mathrm{Re}}^{2}+B_{\mathrm{Im}}^{2}
\end{array}\right) .
$$

Let us note that this Mueller matrix does not depend on the phase shift $\beta$ in Eq. (2). This phase does not influence intensity measurements, but it is crucial to obtain accurate predictions of the phase modulation $\delta$ since it is given by $\delta=\beta+\delta_{M}$, where [5]

$$
\delta_{M}=\arctan \left(\frac{A_{\mathrm{Im}} \cos \left(\varphi_{1}+\varphi_{2}\right)+B_{\mathrm{Im}} \sin \left(\varphi_{1}+\varphi_{2}\right)}{A_{\mathrm{Re}} \cos \left(\varphi_{1}-\varphi_{2}\right)+B_{\mathrm{Re}} \sin \left(\varphi_{1}-\varphi_{2}\right)}\right) .
$$

Here $\delta_{M}$ is the phase contribution that depends on the polarization configuration $\left(\varphi_{1}\right.$ and $\varphi_{2}$ denote the orientation of the input and output polarizers).

The procedure we follow to perform the LCoS full polarimetric characterization is the following:

1. We measure the Mueller matrix $\mathbf{M}$ for the range of addressed gray levels, following a standard procedure as, for instance, presented in [8]. This calibration permits one to predict the intensity modulation $I$ and the degree of polarization versus gray level, by using standard Mueller matrix calculations.

2. We apply the polar decomposition in Eq. (1) to derive diattenuation, depolarization, and retarder contributions.

3. Parameters $A_{\mathrm{Re}}, A_{\mathrm{Im}}, B_{\mathrm{Re}}$, and $B_{\mathrm{Im}}$ are derived by comparing Eq. (3) with the retardance contribution $\mathbf{M}_{\mathbf{R}}$. From these data the phase term $\delta_{M}$ can be predicted using Eq. (4).

4. The phase modulation $\delta$ is measured for a given configuration, and the external mean phase shift is obtained as $\beta=\delta-\delta_{M}$. Once $\beta$ is calibrated, the phase modulation $\delta$ can be predicted for any other polarization configuration.

Once this procedure has been completed, a full polarimetric characterization of the display has been achieved. The Mueller matrix accounts for depolarized light, which is necessary to accurately predict the intensity modulation, while the Jones matrix accounts for the phase modulation. Then the full complex modulation can be predicted and strategies to optimize the diffraction efficiency can be applied.

We use a reflective LCoS display from Holoeye, Model LC-R2500. Experimental calibration of the Mueller matrix was given in [8]. In those results, and also in the new results presented here, we use the wavelength $\lambda=633 \mathrm{~nm}$ from a $\mathrm{He}-\mathrm{Ne}$ laser. Figure 1 shows the intensity, the degree of polarization (DoP), and the phase modulation for the configuration selected to calibrate the phase factor $\beta$. This polarization configuration is chosen since it involves using only polarizers and shows a high intensity transmission in the whole range, thus making phase measurements more accurate. Figure 1(a) shows the intensity modulation and DoP as a function of the gray level. It shows that the amount of depolarized light becomes nonnegligible for gray levels of $\sim 210$. The origin of this depolarization comes from fluctuations in the addressed electrical signal that result in a stronger optical effect for this gray level [8]. The intensity modulation is over 0.5 over the entire gray level range but shows a strong variation, typical in linearly polarized light configurations. The phase modulation has been measured from a two-beam interferometer experiment [8]. It is important to remark that these measurements (both intensity and phase) all correspond to averaged values over several periods of the electrical signal. Figure 1(b) shows the measured phase modulation $(\delta)$ together with the phase term $\delta_{M}$ derived from Eq. (4), and the phase difference $\beta=\delta$ $-\delta_{M}$. Now a prediction of the full modulation can be achieved. In particular we are interested in two configurations: phase-only modulation with $2 \pi$ modulation depth, and amplitude-only modulation. Figure 2 shows the best results we obtain for the phase-only response. We apply an elliptical polarization configuration to obtain a flat intensity modulation [2], and we allow a nonmaximum mean intensity value $(\sim 60 \%)$ to achieve a large modulation depth $[4,11]$. The result in Fig. 2 shows an almost flat intensity modulation accompanied with a phase modulation that reaches $360^{\circ}$, which is a relevant result since it is obtained with a wavelength as long as $\lambda=633 \mathrm{~nm}$ (usually shorter wavelengths are required to obtain this type of response). Figure 3 shows the intensity and phase modulation for a second configuration, again using an elliptical polarization configuration, to obtain an amplitude-mostly response. A monotonously varying intensity modulation with large con- 

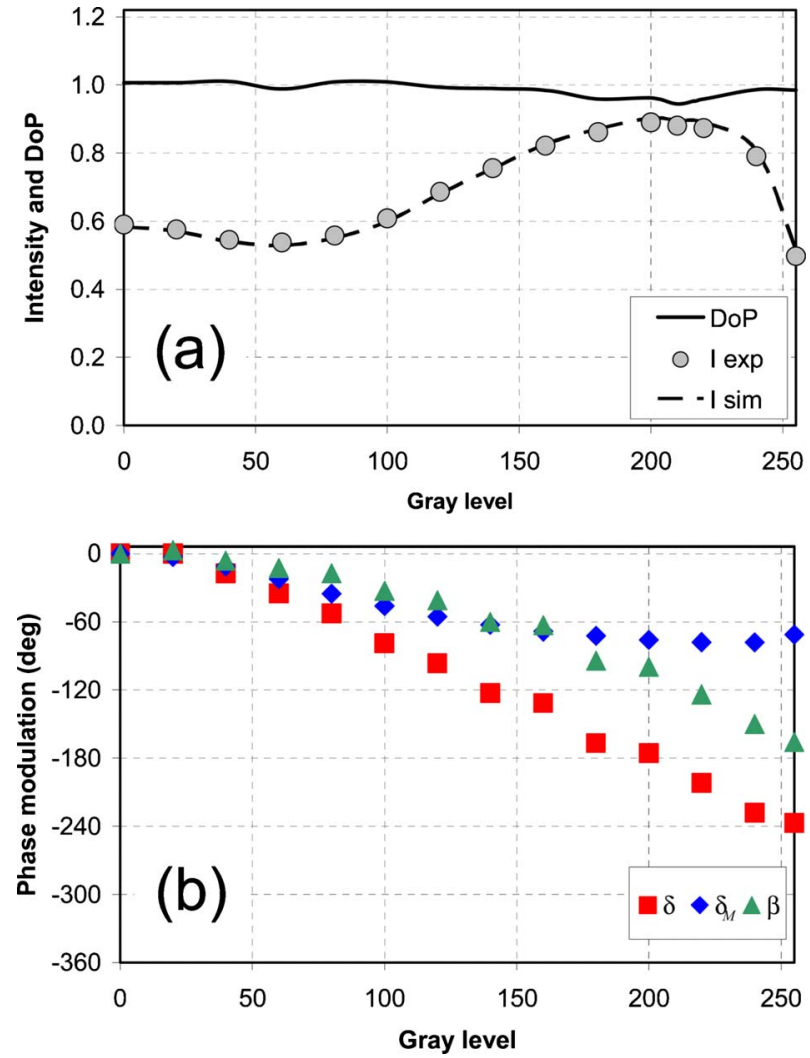

Fig. 1. (Color online) Modulation in the configuration for phase calibration. (a) DoP and intensity modulation. Curves indicate predictions and circles indicate experimental results. (b) Phase modulation terms $\delta, \delta_{M}$, and $\beta$.

trast is obtained with a minimal coupled phase modulation (less than $60^{\circ}$ ). In both cases the agreement between experimental and predicted data is excellent. The DoP is maintained very high in both cases.

In summary, we have presented a technique for a full polarimetric characterization of a LCoS display, which combines the Mueller and Jones formalisms. The polar decomposition is applied to the calibrated Mueller matrix, and the retardance contribution is expressed in terms of the equivalent Jones matrix. The Mueller treatment accounts for depolarization effects, while the Jones treatment accounts for phase modulation. The prediction capability of the inten-

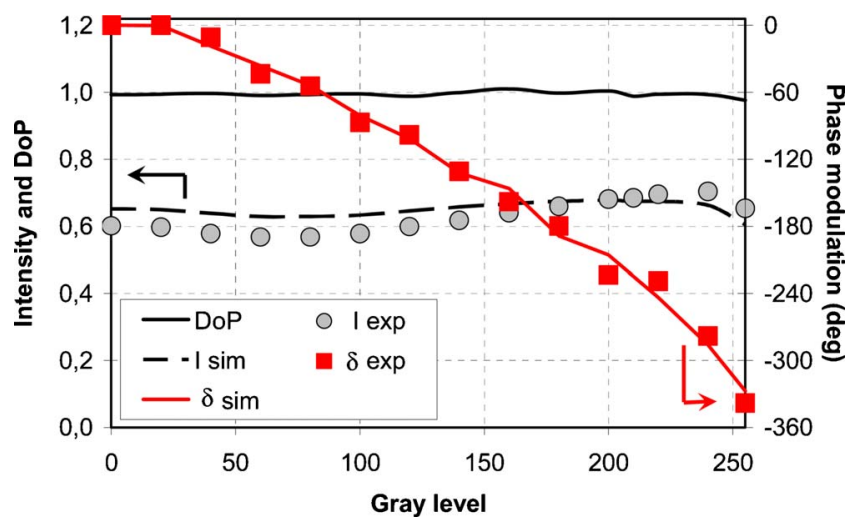

Fig. 2. (Color online) DoP, intensity, and phase modulation in the configuration for phase-only response. Curves indicate predictions, and points indicate experimental data.

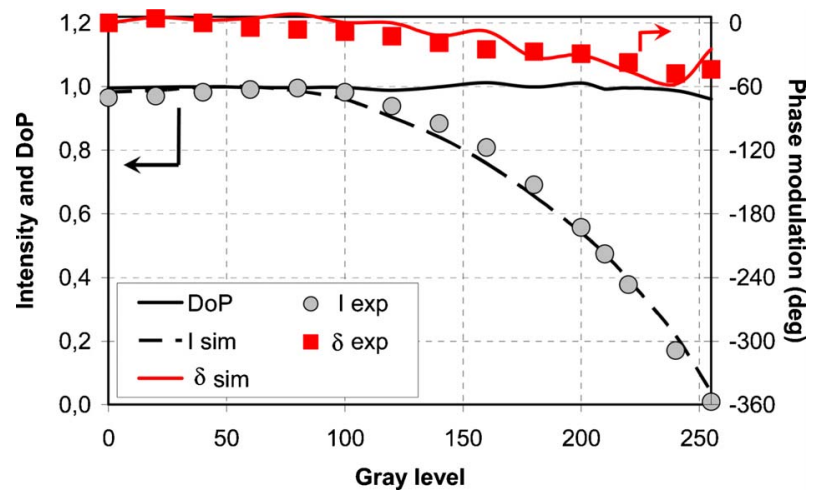

Fig. 3. (Color online) DoP, intensity, and phase modulation in the configuration for amplitude-only response. Curves indicate predictions, and points indicate experimental data.

sity, but also the phase modulation, are the keys to finding the improved configurations presented here for phase-only modulation with $2 \pi$ phase depth or amplitude-mostly modulation. The predictive ability of the proposed procedure has been verified for other polarization configurations (for example, all those shown in [8]) and for other wavelengths [13].

We acknowledge support from the Spanish Ministerio de Educación y Ciencia (FIS2006-13037-C02-01 and 02) and Generalitat de Catalunya (2006PIV00011). C. Iemmi acknowledges support from Universidad de Buenos Aires and CONICET (Argentina).

\section{References}

1. I. Moreno, C. Iemmi, A. Márquez, J. Campos, and M. J. Yzuel, Appl. Opt. 43, 6278 (2004).

2. J. Nicolás, J. Campos, and M. J. Yzuel, J. Opt. Soc. Am. A 19, 1013 (2002).

3. J. Kacperski and M. Kujawinska, Opt. Express 14, 9664 (2006).

4. A. Márquez, C. Iemmi, I. Moreno, J. A. Davis, J. Campos, and M. J. Yzuel, Opt. Eng. (Bellingham) 40, 2558 (2001).

5. I. Moreno, P. Velásquez, C. R. Fernández-Pousa, M. M. Sánchez-López, and F. Mateos, J. Appl. Phys. 94, 3697 (2003).

6. J. L. Pezzaniti, S. C. McClain, R. A. Chipman, and S.-Y. Lu, Opt. Lett. 18, 2071 (1993).

7. J. E. Wolfe and R. A. Chipman, Appl. Opt. 45, 1688 (2006).

8. A. Márquez, I. Moreno, C. Iemmi, A. Lizana, J. Campos, and M. J. Yzuel, Opt. Express 16, 1669 (2008).

9. J. L. Pezzanitti and R. A. Chipman, Opt. Lett. 18, 1567 (1993).

10. V. Durán, J. Lancis, E. Tajahuerce, and Z. Jaroszewicz, J. Appl. Phys. 99, 113101 (2006).

11. J. A. Davis, J. Nicolás, and A. Márquez, Appl. Opt. 41, 4579 (2002).

12. D. Goldstein, Polarized Light, 2nd ed. (Dekker, 2003).

13. A. Lizana, A. Márquez, I. Moreno, C. Iemmi, J. Campos, and M. J. Yzuel, "Wavelength dependence of polarimetric and phase-shift characterization of a liquid crystal on silicon display," J. Eur. Opt. Soc. 3, 08011 (2008). 